\title{
Numerical Analysis of the Chebyshev Collocation Method for Functional Volterra Integral Equations
}

\author{
J. S. AZEVEDO ${ }^{1 *}$, S. M. AFONSO ${ }^{2}$ and M. P. G. DA SILVA ${ }^{3}$
}

Received on January 06, 2020 / Accepted on August 31, 2020

\begin{abstract}
The collocation method based on Chebyshev basis functions, coupled Picard iterative process, is proposed to solve a functional Volterra integral equation of the second kind. Using the Banach Fixed Point Theorem, we prove theorems on the existence and uniqueness solutions in the $L^{2}$-norm. We also provide the convergence and stability analysis of the proposed method, which indicates that the numerical errors in the $L^{2}$-norm decay exponentially, provided that the kernel function is sufficiently smooth. Numerical results are presented and they confirm the theoretical prediction of the exponential rate of convergence.
\end{abstract}

Keywords: functional Volterra integral equation collocation method, Picard iteration.

\section{INTRODUCTION}

In this work we study both theoretical and numerical aspects of solutions of a functional Volterra integral equations of the form

$$
u(x)=g(x)+f\left(x, \int_{a}^{x} \kappa(x, y) u(y) d y\right), \quad x \in(a, b)
$$

where $-\infty<a<y \leq x<b<\infty, f, \kappa$, and $g$ are known functions and $u$ is the solution to be determined. We are able to show that, under certain conditions, the existence and uniqueness of solutions in $L^{2}(a, b)$ of equation (1.1).

At the first moment, we establish a result on the existence and uniqueness of solutions of (1.1) in the function space $L^{2}(a, b)$, whose proof is based on the Banach Fixed Point Theorem. Some authors established criteria for the existence of solutions of nonlinear functional integral equation in

\footnotetext{
*Corresponding author: Juarez S. Azevedo - Email: jdazevedo@ufba.br

${ }^{1}$ Universidade Federal da Bahia - UFBA, Instituto de Ciências, Tecnologia e Inovação, Centro, 42802-721, CamaçariBA, Brazil. - E-mail: jdazevedo@ufba.br https://orcid.org/0000-0002-3641-7604

${ }^{2}$ Universidade Estadual Paulista (UNESP), Instituto de Geociências e Ciências Exatas, 13506-900, Rio Claro-SP, Brasil. - E-mail: s.afonso@unesp.br https://orcid.org/0000-0003-3070-5856

${ }^{3}$ Universidade Federal do Recôncavo da Bahia - UFRB, Centro de Ciências Exatas e Tecnológicas, Centro, 44380-000, Cruz das Almas-BA, Brazil. - E-mail: mpinheiro@ufrb.edu.br https://orcid.org/0000-0003-1801-4227
} 
Banach spaces by using basic fixed point theorems, such as $[8,10,11,12,13]$, for instance. However, the conditions about existence and uniqueness were used depending on the Carathéodorytype conditions of the kernel, which are not required in this work. Essentially here, classical inequalities used for $L^{2}(a, b)$-functions, such as Hölder inequality and Minkowski inequality, combined with Banach Fixed Point Theorem, allow us to guarantee the existence and uniqueness of solutions of (1.1).

In the numerical part, we consider the Chebyshev collocation method $[4,5,6]$ and the Picard iterative process [14] for the numerical solution of nonlinear integral equation (1.1). Our tasks in this step are to show that the approximate solution converges, under particular conditions, to an exact solution in $L^{2}(a, b)$, and to analysis the rate of this convergence.

Although there is a rich literature of Chebyshev collocation methods related to Volterra integral equations, see $[3,16,17,19]$, the convergence analysis of the functional Volterra integral equation (1.1) is a new study for this theory. Such methodology was implemented based on the Chebyshev polynomials of degree $N$, which has as main advantage an infinite convergence rate (in terms of the order of accuracy), since it uses high degree polynomials as shape functions. This also allows that the number of collocation points inside each element can be increased, which guarantees a better approximation of the solution. In the case of the iterative process, the Picard iteration method was chosen because of its simple computational implementation, besides calculating the nonlinear problem by finding successive approximations to the solution by starting with the zeroth approximation. Numerical results validate the proposed scheme and reveal an exponential convergence when we deal with regular functions, regardless of the iterative procedure used. Similar results were obtained by others authors from error analysis for the collocation method [21] and the Galerkin spectral method [2,20] involving Legendre and Jacobi basis functions, respectively. This methodology can be disseminated to other integro-differential equations. In addition, this methodology can be disseminated to other integro-differential equations. Note that the conditions presented in the text allow slight modifications to be made so that several classes of integral equations are inserted in this context.

This paper is organized as follows. In Section 2, we highlight some preliminary concepts, while in Section 3 we present a theorem about the existence and uniqueness of solutions in the space $L^{2}(a, b)$. In Section 4, we outline Chebyshev collocation method to solve nonlinear integral equations. The convergence analysis of the methods to show their order of convergence is established in Section 5. In Section 6, numerical examples are provided to demonstrate the effectiveness of the proposed method.

\section{PRELIMINARY ASSUMPTIONS}

Here, we introduce some notations and concepts that will be used throughout in this paper. We begin our study presenting the Hilbert space $L^{2}(a, b)$ endowed with the usual inner product and its induced norm

$$
(u, v)=\int_{a}^{b} u(x) v(x) d x, \quad\|v\|_{L^{2}(a, b)}=(v, v)^{1 / 2},
$$


along with the space $H^{m}(a, b)=\left\{v: D^{k} v \in L^{2}(a, b), 0 \leq k \leq m, m \geq 1\right\}$ equipped with the norm

$$
|v|_{m}=\left(\sum_{k=1}^{m}\left\|D^{k} v\right\|_{L^{2}(a, b)}^{2}\right)^{\frac{1}{2}}
$$

where $D^{k} v=\frac{\partial^{k} v}{\partial x^{k}}$.

In order to rewrite the nonlinear integral equation (1.1) in the form of a functional operator, let us define $H$ by

$$
H=F K,
$$

where $K$ is an integral linear operator expressed by

$$
(K u)(x)=\int_{a}^{x} \kappa(x, y) u(y) d y, \quad \text { with } \kappa:(a, b) \times(a, b) \longrightarrow \mathbb{R},
$$

and $F$ is the superposition operator, associated to $f$, defined by $(F u)(x)=f(x, u(x))$ for each $x \in(a, b)$, i.e.,

$$
(H u)(x)=(F K u)(x)=f\left(x, \int_{a}^{x} \kappa(x, y) u(y) d y\right) \quad \text { for } x \in(a, b) .
$$

We define an operator $A$ by

$$
A u=g+H u .
$$

Now, we are going to show the existence and uniqueness of solutions of equation (2.2). We consider, in the next section, that the result about existence is based on determining a fixed point for the operator $A$. For this end, we will consider certain conditions on the functions $g, f$ and $\kappa$, as follows:

(A1) $g \in L^{2}(a, b)$.

(A2) $\kappa \in L^{2}((a, b) \times(a, b))$, i. e., $\|\kappa\|_{2}:=\left(\int_{a}^{b} \int_{a}^{b}|k(x, y)|^{2} d x d y\right)^{1 / 2}<\infty$.

(A3) $f:(a, b) \times \mathbb{R} \longrightarrow \mathbb{R}$ satisfies the Carathéodory conditions and there are a constant $\tau_{1}>0$ and a nonnegative function $\theta_{1} \in L^{2}(a, b)$ such that

$$
|f(x, y)| \leq \theta_{1}(x)+\tau_{1}|y|, \quad x \in(a, b), y \in \mathbb{R}
$$

(A4) $f$ is Lipschitz with respect to the second variable with Lipschitz constant $\tau_{2}>0$, i.e.,

$$
|f(x, u)-f(x, v)| \leq \tau_{2}|u-v|,
$$

for all $x \in(a, b)$ and $u, v \in \mathbb{R}$.

(A5) The kernel $\kappa$ and the constant $\tau_{2}$ satisfy

$$
\tau_{2}\|\kappa\|_{2}<1
$$




\section{EXISTENCE AND UNIQUENESS}

In this section, we present a result on the existence and uniqueness of solutions for the functional Volterra integral equation (1.1) in $L^{2}(a, b)$. At first, we introduce an important preliminary result.

Lemma 3.1. If the functions $g, f$, and $\kappa$ satisfy conditions $(\mathbf{A 1})$ to $(\mathbf{A 3})$, then A applies $L^{2}(a, b)$ on itself, i.e.,

$$
\forall u \in L^{2}(a, b) \quad \Longrightarrow \quad A u \in L^{2}(a, b)
$$

Proof. It suffices to show that the operator $H$ applies $L^{2}(a, b)$ on itself. Given $u \in L^{2}(a, b)$, using the assumption (A3) we have

$$
\begin{aligned}
\|H u\|_{2} & =\left(\int_{a}^{b}|F(K u)(x)|^{2} d x\right)^{1 / 2} \\
& =\left(\int_{a}^{b}|f(x,(K u)(x))|^{2} d x\right)^{1 / 2} \\
& \leq\left(\int_{a}^{b}\left|\theta_{1}(x)+\tau_{1}\right|(K u)(x) \|^{2} d x\right)^{1 / 2} .
\end{aligned}
$$

Applying Minkowski inequality, we obtain

$$
\begin{aligned}
\|H u\|_{2} & \leq\left(\int_{a}^{b}\left|\theta_{1}(x)\right|^{2} d x\right)^{1 / 2}+\left(\int_{a}^{b}\left|\tau_{1}\right|(K u)(x) \|^{2} d x\right)^{1 / 2} \\
& =\left\|\theta_{1}\right\|_{2}+\tau_{1}\left(\int_{a}^{b}\left|\int_{a}^{x} \kappa(x, y) u(y) d y\right|^{2} d x\right)^{1 / 2} \\
& \leq\left\|\theta_{1}\right\|_{2}+\tau_{1}\left(\int_{a}^{b}\left(\int_{a}^{b}|\kappa(x, y) u(y)| d y\right)^{2} d x\right)^{1 / 2}
\end{aligned}
$$


Then, applying Hölder inequality, we get

$$
\begin{aligned}
\|H u\|_{2} & \leq\left\|\theta_{1}\right\|_{2}+\tau_{1}\left\{\int_{a}^{b}\left[\left(\int_{a}^{x}|\kappa(x, y)|^{2} d y\right)^{1 / 2}\left(\int_{a}^{x}|u(y)|^{2} d y\right)^{1 / 2}\right]^{2} d x\right\}^{1 / 2} \\
& \leq\left\|\theta_{1}\right\|_{2}+\tau_{1}\left\{\int_{a}^{b}\left[\left(\int_{a}^{b}|\kappa(x, y)|^{2} d y\right)^{1 / 2}\left(\int_{a}^{b}|u(y)|^{2} d y\right)^{1 / 2}\right]^{2} d x\right\}^{1 / 2} \\
& =\left\|\theta_{1}\right\|_{2}+\tau_{1}\left\{\int_{a}^{b} \int_{a}^{b}|\kappa(x, y)|^{2} d y d x\right\}^{1 / 2}\|u\|_{2} \\
& =\left\|\theta_{1}\right\|_{2}+\tau_{1}\left\{\int_{a}^{b} \int_{a}^{b}|\kappa(x, y)|^{2} d x d y\right\}^{1 / 2}\|u\|_{2} \\
& =\left\|\theta_{1}\right\|_{2}+\tau_{1}\|\kappa\|_{2}\|u\|_{2} .
\end{aligned}
$$

Therefore, $\|H u\|_{2}<\infty$ and $H u \in L^{2}(a, b)$.

The existence and uniqueness of the solution of the functional Volterra integral equation (1.1) is guaranteed by the next result, whose proof is based on the Banach Fixed Point Theorem. We refer the reader to [9], for more details on this fixed point theorem.

Theorem 3.1. Consider the nonlinear functional Volterra integral equation (1.1), where $g, f$, and $\kappa$ satisfy conditions (A1) to (A5). Then, there exists a unique function $u \in L^{2}(a, b)$ such that $u=A u$.

Proof. We have from Lemma 3.1 that $A$ maps $L^{2}(a, b)$ to $L^{2}(a, b)$. From the Banach Fixed Point Theorem, it remains to prove that $A$ is a contraction. Indeed, we have from condition (A4) that, for all $x \in(a, b)$,

$$
\begin{aligned}
|A u(x)-A v(x)| & =|f(x,(K u)(x))-f(x,(K v)(x))| \\
& \leq \tau_{2}|(K u)(x)-(K v)(x)| .
\end{aligned}
$$

Thus,

$$
\|A u-A v\|_{2} \leq \tau_{2}\|K(u-v)\|_{2} \leq \tau_{2}\|\kappa\|_{2}\|u-v\|_{2}
$$

Since $\tau_{2}\|k\|_{2}<1$ by condition (A5), we get the desired result.

\section{NUMERICAL APPROXIMATION}

Let us suppose that

$$
u(x)=\sum_{j=1}^{\infty} a_{j} \phi_{j}(x)
$$


and $u_{N}(x)$ represents its respective approximation, i.e.,

$$
u(x) \approx u_{N}(x)=\sum_{j=1}^{N} a_{j} \phi_{j}(x)=u_{N} \Phi(x)
$$

where $u_{N}=\left(a_{1}, a_{2}, \ldots, a_{N}, 0, \ldots\right)^{T}$ and $\Phi(x)=\left(\phi_{1}(x), \phi_{2}(x), \ldots, \phi_{N}(x), 0, \ldots\right)^{T}$ are the shifted Chebyshev polynomials of degree $N$ defined on the interval $(a, b)$.

Substituting the relation (4.1) in (1.1), we get

$$
u_{N} \Phi(x)=g(x)+f\left(x, \int_{a}^{x} \kappa(x, y) u_{N} \Phi(y) d y\right) .
$$

Following Driscoll [4], since $x=\left(x_{1}, x_{2}, \ldots, x_{N}\right)^{T}$ are the $N$ Chebyshev nodes and $K W u_{N} \Phi(x)$ is the matrix that contains values of the approximated integral (4.2) at the same nodes, we have

$$
\begin{aligned}
& K W u_{N} \Phi(x) \\
& =\left[\begin{array}{cccc}
w_{11} \kappa\left(x_{1}, x_{1}\right) & w_{12} \kappa\left(x_{1}, x_{2}\right) & \ldots & w_{1 N} \kappa\left(x_{1}, x_{N}\right) \\
w_{21} \kappa\left(x_{2}, x_{1}\right) & w_{22} \kappa\left(x_{2}, y_{2}\right) & \ldots & w_{2 N} \kappa\left(x_{2}, x_{N}\right) \\
\vdots & \vdots & \ddots & \vdots \\
w_{N 1} \kappa\left(x_{N}, x_{1}\right) & w_{N 2} \kappa\left(x_{N}, x_{2}\right) & \cdots & w_{N N} \kappa\left(x_{N}, x_{N}\right)
\end{array}\right] u_{N} \Phi(x),
\end{aligned}
$$

where

$$
u_{N} \Phi(x)=\left(u_{N} \Phi\left(x_{1}\right), u_{N} \Phi\left(x_{2}\right), \ldots, u_{N} \Phi\left(x_{N}\right)\right)^{T},
$$

and $\left\{w_{i j}\right\}$ for each fixed $i$ are the quadrature weights relative to integration interval $\left(a, x_{i}\right]$, with $\left(a, x_{i}\right] \subset(a, b)$. We refer the reader to Trif and Ionescu [18] for more details on the numerical implementation of this integral. Substituting the expression (4.3) into (4.2), we obtain

$$
u_{N} \Phi(x)=g(x)+f\left(x, K W u_{N} \Phi(x)\right),
$$

such that $g(x)=\left[g\left(x_{1}\right), g\left(x_{2}\right), \ldots, g\left(x_{N}\right)\right]^{T}$.

Due to the nonlinearity of this expression, we resort to an iterative procedure. In this case, we choose the Picard iterative method that consists of determining a sequence $\left(u_{N}^{(k+1)}\right)$ in which the following relation of recurrence holds

$$
u_{N}^{(k+1)} \Phi(x)=g(x)+f\left(x, K W u_{N}^{(k)} \Phi(x)\right), \quad k \geq 0 .
$$

The procedure is concluded provided that one of the following criteria is satisfied

$$
k>k_{\max } \quad \text { or } \quad r_{k}=\left\|u^{(k)}-u^{(k-1)}\right\|_{2}<t_{0} l_{1} .
$$

Once we get the solution vector $u_{N}^{(k)}$, we have to relate it to the basis functions $\left\{\phi_{i}\right\}_{i=1}^{N}$ as follows

$$
u_{N}^{(k)} \Phi(x)=\sum_{i=1}^{N} a_{i}^{(k)} \phi_{i}(x)=u_{N}^{(k)}(x)
$$


Finally, we obtain a solution $u_{N}^{(k)}$ that must satisfy

$$
u_{N}^{(k)}(x)=g(x)+f\left(x, \int_{a}^{x} \kappa(x, y) u_{N}^{(k)}(y) d y\right), \quad \forall x \in(a, b) .
$$

In the sequel, we present error estimates for the space discretization error of this functional integral equation.

\section{CONVERGENCE ANALYSIS AND STABILITY}

We provide a convergence analysis and a stability criterion of the proposed method for the numerical solution of the functional nonlinear Volterra integral equation (1.1). In our subsequent analysis, some definitions and results are needed.

Firstly, we introduce the orthogonal projection $\Pi_{N}: L^{2}(a, b) \rightarrow \mathscr{P}_{N}$ which is a mapping such that for any $v \in L^{2}(a, b)$ satisfies

$$
\left(v-\Pi_{N} v, \phi\right)=0, \quad \forall \phi \in \mathscr{P}_{N},
$$

where $\mathscr{P}_{N}$ is the space of all algebraic polynomials of degree up to $N$. Concerning the truncation error of a Chebyshev interpolation, the following estimate holds (for more details, see [1,3])

$$
\left\|u-\Pi_{N} u\right\|_{2} \leq \widetilde{C} N^{-m}|u|_{m},
$$

for all $u \in H^{m}(a, b)$, with $m \geq 0$, where $|\cdot|_{m}$ was defined in (2.1) and $\widetilde{C}>0$.

In the sequel, we shall apply the Gronwall inequality (which can be found in [7]): Suppose $E(x)$ is a non-negative, locally integrable function defined on $(a, b)$, satisfying

$$
E(x) \leq G(x)+\int_{a}^{x} E(y) d y .
$$

such that $G(x) \geq 0$. Then, there exists a positive constant $C$ such that

$$
\|E\|_{2} \leq C\|G\|_{2}
$$

The next result is devoted to providing a convergence analysis for the numerical scheme, showing that the rate of convergence is exponential.

Theorem 5.1. Let $u_{N}(\cdot)$ be the collocation approximation (4.1) to the exact solution $u(\cdot)$ of the functional nonlinear Volterra integral equation (1.1) and consider $e_{N}(x)=u(x)-u_{N}(x)$ the error function and

$$
\left(F K u_{N}\right)(x)=f\left(x, \int_{a}^{x} \kappa(x, y) u_{N}(y) d y\right) .
$$

If the functions $g(\cdot), f(\cdot, \cdot)$ and $\kappa(\cdot, \cdot)$ are such that $g \in H^{\left(k_{1}\right)}(a, b), F K u_{N} \in H^{\left(k_{2}\right)}(a, b), \kappa \in$ $H^{\left(k_{3}\right)}((a, b) \times(a, b))$, where $k_{i} \geq 0, i=1,2,3$, and $f(\cdot, \cdot)$ satisfies assumption (A4), then we have

$$
\left.\left\|e_{N}\right\|_{2} \leq\left.\mu\left(N^{-k_{1}}|g|_{k_{1}}+N^{-k_{2}} \mid\left(F K u_{N}\right)(x)\right)\right|_{k_{2}}+N^{-k_{3}}|\kappa|_{k_{3}}\left\|u_{N}\right\|_{2}\right),
$$


provided that $N$ is sufficiently large and $\mu$ is a constant independent of $N$.

Proof. The ideas of this proof were borrowed from Ansari and Mokhtary [1]. Let

$$
u_{N}(x)=\Pi_{N} g(x)+\Pi_{N} f\left(x, \int_{a}^{x} \Pi_{N} \kappa(x, y) u_{N}(y) d y\right),
$$

and consider the difference

$$
\begin{aligned}
e_{N}(x) & =g(x)-\Pi_{N} g(x) \\
& +f\left(x, \int_{a}^{x} \kappa(x, y) u(y) d y\right)-\Pi_{N} f\left(x, \int_{a}^{x} \Pi_{N} \kappa(x, y) u_{N}(y) d y\right) \\
& =(F K u)(x)-\left(F K u_{N}\right)(x)+J_{0}+J_{1}+J_{2},
\end{aligned}
$$

such that

$$
\begin{gathered}
J_{0}=g(x)-\Pi_{N} g(x) \\
J_{1}=f\left(x, \int_{a}^{x} \kappa(x, y) u_{N}(y) d y\right)-\Pi_{N} f\left(x, \int_{a}^{x} \kappa(x, y) u_{N}(y) d y\right)
\end{gathered}
$$

and

$$
J_{2}=\Pi_{N}\left(f\left(x, \int_{a}^{x} \kappa(x, y) u_{N}(y) d y\right)-f\left(x, \int_{a}^{x} \Pi_{N} \kappa(x, y) u_{N}(y) d y\right)\right) .
$$

Since $f$ is Lipschitz in the second variable by (A4), we have

$$
\begin{aligned}
e_{N}(x) & =(F K u)(x)-\left(F K u_{N}\right)(x)+J_{0}+J_{1}+J_{2} \\
& \leq \tau_{2}\left|\int_{a}^{x} \kappa(x, y) e_{N}(y) d y\right|+J_{0}+J_{1}+J_{2} .
\end{aligned}
$$

Anchored by the Gronwall Inequality, we can affirm that there exists $C>0$ such that

$$
\|e\|_{2} \leq C\left(\left\|J_{0}\right\|_{2}+\left\|J_{1}\right\|_{2}+\left\|J_{2}\right\|_{2}\right)
$$

Employing inequality (5.1), an estimate for $J_{0}$ is given below:

$$
\left\|J_{0}\right\|_{2} \leq \widetilde{C}_{1} N^{-k_{1}}|g|_{k_{1}},
$$

since $g \in H^{\left(k_{1}\right)}(a, b)$, where $\widetilde{C}_{1}>0$. Using again (5.1), we get

$$
\left\|J_{1}\right\|_{2} \leq \widetilde{C}_{2} N^{-k_{2}}\left|\left(F K u_{N}\right)(x)\right|_{k_{2}}
$$


provided $F K u_{N} \in H^{\left(k_{2}\right)}(a, b)$, where $\widetilde{C}_{2}>0$. Since $\Pi_{N}$ is an orthogonal projection, we have $\left\|\Pi_{N}\right\|_{2}=1$. Moreover, seeing that $f$ satisfies (A4), we obtain

$$
\begin{aligned}
\left\|J_{2}\right\|_{2} & \leq\left\|f\left(x, \int_{a}^{x} \kappa(x, y) u_{N}(y) d y\right)-f\left(x, \int_{a}^{x} \Pi_{N} \kappa(x, y) u_{N}(y) d y\right)\right\|_{2} \\
& \leq \tau_{2}\left\|\int _ { a } ^ { x } \left|\kappa(x, y)-\Pi_{N} \kappa(x, y)\left\|u_{N}(y) \mid d y\right\|_{2}\right.\right. \\
& =\tau_{2} \sqrt{\int_{a}^{b}\left(\int_{a}^{x}\left|\kappa(x, y)-\Pi_{N} \kappa(x, y) \| u_{N}(y)\right| d y\right)^{2} d x} \\
& \leq \tau_{2} \sqrt{\int_{a}^{b}\left(\int_{a}^{b}\left|\kappa(x, y)-\Pi_{N} \kappa(x, y) \| u_{N}(y)\right| d y\right)^{2} d x} \\
& \leq \tau_{2} \sqrt{\int_{a}^{b} \int_{a}^{b}\left(\kappa(x, y)-\Pi_{N} \kappa(x, y)\right)^{2} d y \int_{a}^{b}\left(u_{N}(y)\right)^{2} d y d x} \\
& \leq L_{1} \widetilde{C}_{3} N^{-k_{3}}|\kappa|_{k_{3}}\left\|u_{N}\right\|_{2},
\end{aligned}
$$

since $\kappa \in H^{\left(k_{3}\right)}((a, b) \times(a, b))$, where $L_{1}$ depends on $b-a$ and $\widetilde{C}_{3}>0$.

Let $\widetilde{C}=\max \left\{\widetilde{C}_{1}, \widetilde{C}_{2}, L_{1} \cdot \widetilde{C}_{3}\right\}$. Then,

$$
\begin{gathered}
\left\|J_{0}\right\|_{2} \leq \widetilde{C} N^{-k_{1}}|g|_{k_{1}}, \quad\left\|J_{1}\right\|_{2} \leq \widetilde{C} N^{-k_{2}}\left|\left(F K u_{N}\right)(x)\right|_{k_{2}} \quad \text { and } \\
\left\|J_{2}\right\|_{2} \leq \widetilde{C} N^{-k_{3}}|\kappa|_{k_{3}}\left\|u_{N}\right\|_{2} .
\end{gathered}
$$

Combining (5.8) and (5.9) in (5.4), we obtain

$$
\|e\|_{2} \leq C\left(\widetilde{C} N^{-k_{1}}|g|_{k_{1}}+\widetilde{C} N^{-k_{2}}\left|\left(F K u_{N}\right)(x)\right|_{k_{2}}+\widetilde{C} N^{-k_{3}}|\kappa|_{k_{3}}\left\|u_{N}\right\|_{2}\right) .
$$

Taking $\mu=C \cdot \widetilde{C}$, we derive that

$$
\left.\left\|e_{N}\right\|_{2} \leq\left.\mu\left(N^{-k_{1}}|g|_{k_{1}}+N^{-k_{2}} \mid\left(F K u_{N}\right)(x)\right)\right|_{k_{2}}+N^{-k_{3}}|\kappa|_{k_{3}}\left\|u_{N}\right\|_{2}\right),
$$

which completes the proof.

In the following theorem we provide a stability analysis for the solution of (1.1) in the $L^{2}$-norm.

Theorem 5.2. (Stability) Assume that conditions (A4) and (A5) are satisfied. In addition, suppose $\tilde{u}$ and $\tilde{g}$ are the small perturbations of $u_{N}$ and $g$ in (5.3), respectively. Then,

$$
\|\tilde{u}\|_{2} \leq \frac{1}{1-\tau_{2}\|\kappa\|_{2}}\|\tilde{g}\|_{2}
$$


Proof. To prove this result, we followed again the steps from Ansari and Mokhtary [1]. Using eq. (5.3), we can infer that $u_{N}+\tilde{u}$ satisfies the following equation

$$
u_{N}+\tilde{u}=\Pi_{N}(g+\tilde{g})+\Pi_{N} f\left(x, \int_{a}^{x} \Pi_{N} \kappa(x, y)\left(u_{N}+\tilde{u}\right)(y) d y\right) .
$$

Calculating the difference between (5.11) and (5.3) we obtain

$$
\tilde{u}=\Pi_{N} \tilde{g}+\Pi_{N} f\left(x, \int_{a}^{x} \Pi_{N} \kappa(x, y)\left(u_{N}+\tilde{u}\right)(y) d y\right)-\Pi_{N} f\left(x, \int_{a}^{x} \Pi_{N} \kappa(x, y) u_{N}(y) d y\right) .
$$

Since $f$ is Lipschitz in the second variable by assumption (A4), we get

$$
\begin{aligned}
& \Pi_{N} f\left(x, \int_{a}^{x} \Pi_{N} \kappa(x, y)\left(u_{N}+\tilde{u}\right)(y) d y\right)-\Pi_{N} f\left(x, \int_{a}^{x} \Pi_{N} \kappa(x, y) u_{N}(y) d y\right) \\
& \leq \tau_{2}\left|\int_{a}^{x} \Pi_{N} \kappa(x, y) \tilde{u}(y) d y\right| .
\end{aligned}
$$

Now, since $\Pi_{N}$ is an orthogonal projection $\left(\left\|\Pi_{N}\right\|_{2}=1\right)$, we derive

$$
\begin{aligned}
\|\tilde{u}\|_{2} & \leq\|\tilde{g}\|_{2}+\tau_{2}\left\|\Pi_{N} \kappa\right\|_{2}\|\tilde{u}\|_{2} \\
& \leq\|\tilde{g}\|_{2}+\tau_{2}\|\kappa\|_{2}\|\tilde{u}\|_{2},
\end{aligned}
$$

whence it follows the result.

\section{NUMERICAL EXPERIMENTS}

In this section we present two numerical examples to verify the accuracy of the method and the effectiveness of applying the Chebyshev polynomial bases of degree $N$. In theses examples, we also consider collocation method with Legendre polynomial bases of degree $N$.

In order to ensure the accuracy in the Picard iterative schemes, we admit $t_{0} l_{1}=1 e-12$ and $k_{\max }=1000$ in the stopping criterion defined in (4.5).

The error is estimated using the relative error:

$$
e_{r}=\frac{\left\|u-u_{N}\right\|_{2}}{\|u\|_{2}}=\frac{\left\|e_{N}\right\|_{2}}{\|u\|_{2}}
$$

The calculation of the order of accuracy $p_{N}$ uses the expression (see Ansari [1]):

$$
p_{N}=\log _{2}\left(\frac{\left\|e_{N}\right\|_{2}}{\left\|e_{N / 2}\right\|_{2}}\right) \text {. }
$$

In all experiments we take as initial guess the constant function $u^{(0)}(x)=0$ for the Picard iterations. 


\subsection{Example 1}

Consider the following functional Volterra integral equation

$$
u(x)=g(x)+f\left(x, \int_{0}^{x} \kappa(x, y) u(y) d y\right), \quad x \in(0, \pi / 2),
$$

with kernel

$$
\kappa(x, y)=\sin (y-x) .
$$

Here, the function $f$ is expressed by $f(x, y)=\sin (y)$ and $g$ is defined by

$$
g(x)=\exp (x)-\sin ((-\exp (x)+(\cos (x)+\sin (x))) / 2) .
$$

Simple calculations show that $f, g$, and $\kappa$ satisfy all hypotheses from Theorem 3.1 , with $\tau_{1}=1$, $\theta_{1}(x)=0$ for all $x \in \mathbb{R}, \tau_{2}=1$, and $\tau_{2}\|\kappa\|_{2} \approx 0.8565<1$. Therefore, the solution of (6.1) exists and it is unique, given by

$$
u(x)=\exp (x), \quad \text { for } x \in(0, \pi / 2) .
$$

Let $u_{N}(\cdot)$ be the collocation approximation to the exact solution $u(\cdot)$ of the functional nonlinear Volterra integral equation (6.1). According to (4.4), equation (6.1) can be rewritten in the following vector form

$$
u_{N}^{(k+1)} \Phi(x)=g(x)+\sin \left(K W u_{N}^{(k)} \Phi(x)\right), \quad k \geq 0,
$$

such that

$$
u_{N}^{(k)} \Phi(x)=\left[u_{N}^{(k)}\left(x_{1}\right), u_{N}^{(k)}\left(x_{2}\right), \ldots, u_{N}^{(k)}\left(x_{N}\right)\right]^{T}
$$

and

$$
\begin{aligned}
& K W \\
& =\left[\begin{array}{cccc}
0 & w_{12} \sin \left(x_{2}-x_{1}\right) & \cdots & w_{1 N} \sin \left(x_{N}-x_{1}\right) \\
w_{21} \sin \left(x_{1}-x_{2}\right) & 0 & \cdots & w_{2 N} \sin \left(x_{N}-x_{2}\right) \\
\vdots & \vdots & \ddots & \vdots \\
w_{N 1} \sin \left(x_{1}-x_{N}\right) & w_{N 2} \sin \left(x_{2}-x_{N}\right) & \cdots & 0
\end{array}\right] .
\end{aligned}
$$

In Figures 1a-1b, we plot numerical errors obtained in terms of the number of approximation $N$ in semi-log representation and we observe an approximately linear behavior which stabilizes at determined values of $N$ for each iteration $k$. Very few iterations are enough so that the relative error to be approximately $10^{-15}$, in particular, when $k=20$, we obtain $e_{r} \leq 10^{-15}$. This is confirmed in Figs. 1c-1d, which present the exponential decay of the error (see Theorem 5.1).

The convergence orders using orthogonal bases of Chebyshev and Legendre were illustrated in Table 1 and grow exponentially with $N$, which confirms our theoretical predictions. Note that for a reasonable value of $N$ there is a sharp drop in the convergence order, since the error size has reached the machine epsilon. 


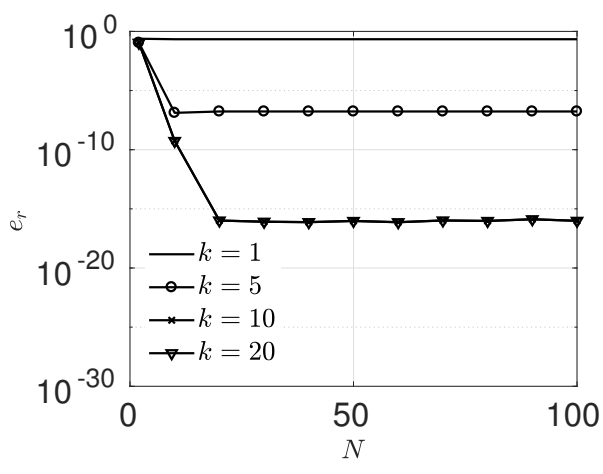

(a) Chebyshev

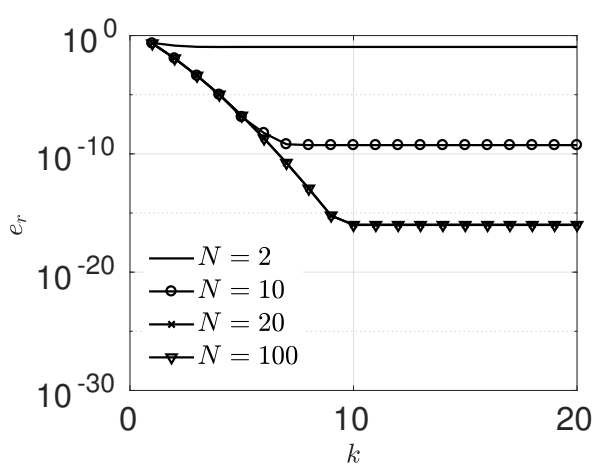

(c) Chebyshev

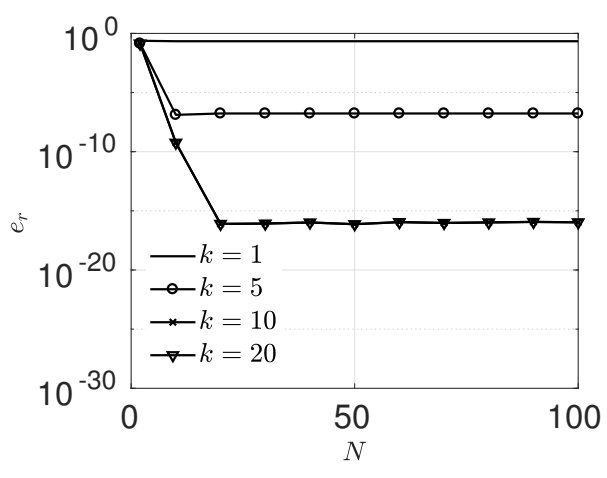

(b) Legendre

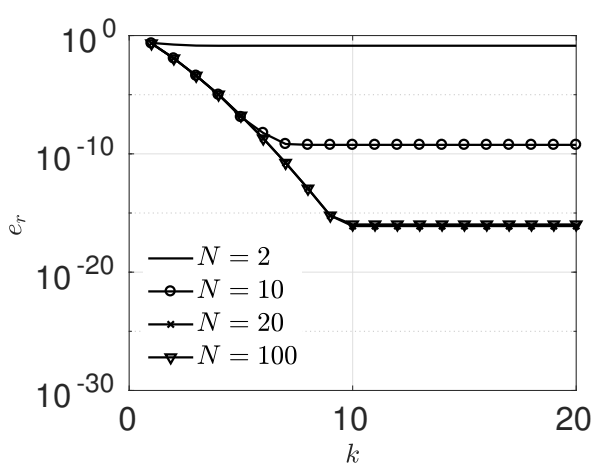

(d) Legendre

Figure 1: The relative error in terms of degree $N(\mathrm{a}-\mathrm{b})$ and the number of iterations (c-d) corresponding to Example 1 for Chebyshev and Legendre bases.

\subsection{Example 2}

In this example, let us consider the following functional Volterra integral equation

$$
u(x)=(\sin (x)-x)-f\left(x, \int_{0}^{x} \exp \left(-\lambda^{2}(x-y)^{2}\right) u(y) d y\right), \quad \forall x \in(0, \pi / 4),
$$

where the parameter $\lambda$ defines the length scale, $f(x, y)=\sin (y), g(x)=\sin (x)-x$, and $\kappa(x, y)=$ $\exp \left(-\lambda^{2}(x-y)^{2}\right)$.

In this example, the exact solution is not known. However, equation (6.4) admits a unique solution, since all conditions from Theorem 3.1 are satisfied, with $\tau_{1}=1$ and $\theta_{1}(x)=0$ for all $x \in \mathbb{R}$. For instance, if $\lambda=0.1$, conditions (A4) and (A5) are satisfied with $\tau_{2}=1$ and $\tau_{2}\|\kappa\|_{2} \approx 0.7848<1$.

Now, we study how the parameter $\lambda$ affects the relative error $e_{r}$. According to Figure 2, a large value of $\lambda$ favors a large error due to small smoothing, while a small value of $\lambda$ stabilizes the 


\begin{tabular}{ccc}
\hline $\mathrm{N}$ & Chebyshev basis & Legendre basis \\
\hline 4 & 6.1642 & 6.6870 \\
8 & 13.9638 & 13.6007 \\
12 & 25.0964 & 25.1034 \\
20 & 22.3432 & 22.8064 \\
100 & 0.0418 & 0.5310 \\
\hline
\end{tabular}

Table 1: Convergence order with respect to Chebyshev and Legendre basis considering $k=20$ corresponding to Example 1.

error in small values due to a high degree of smoothing. In this case, the exact solution can behave as a constant when $\lambda \rightarrow 0$.

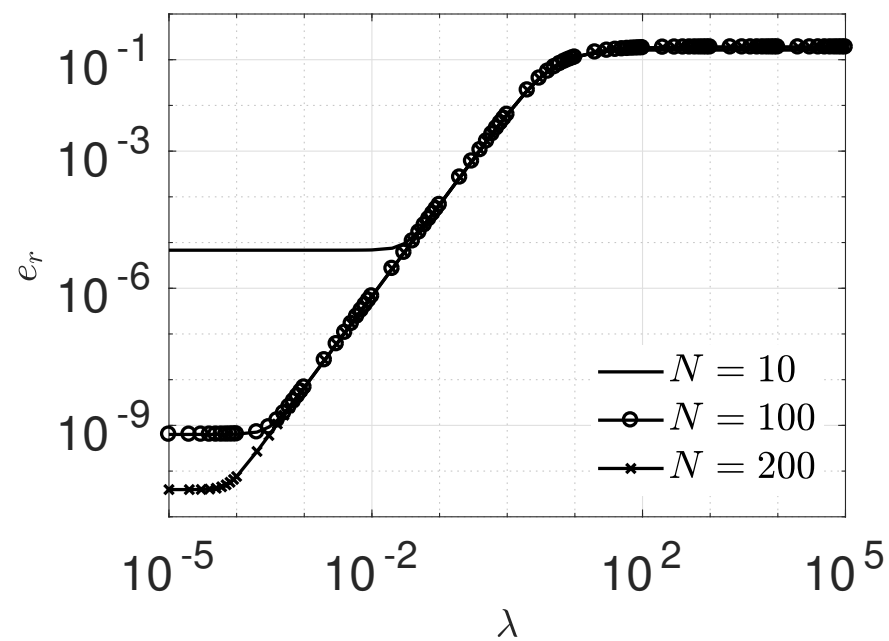

Figure 2: Relative error $e_{r}$ in terms of the parameter $\lambda$, with $N=10,100$ and 200 considering Chebyshev functions.

Figures 3a-3a and 3c-3d show the behavior of the relative error in terms of $N$ and $k$, respectively, considering Chebyshev functions of first kind and the Legendre polynomial. As in the previous example, we can observe the convergence in the $L^{2}$-norm in terms of $N$, where no more than 20 iterations and 100 integration points were enough to get the relative error close to $10^{-9}$ in both experiments. 


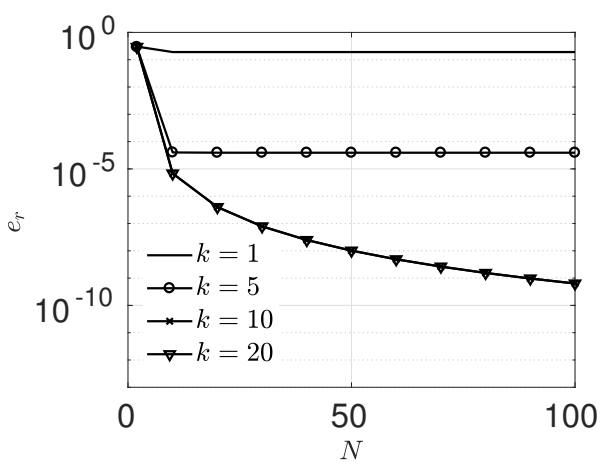

(a) Chebyshev

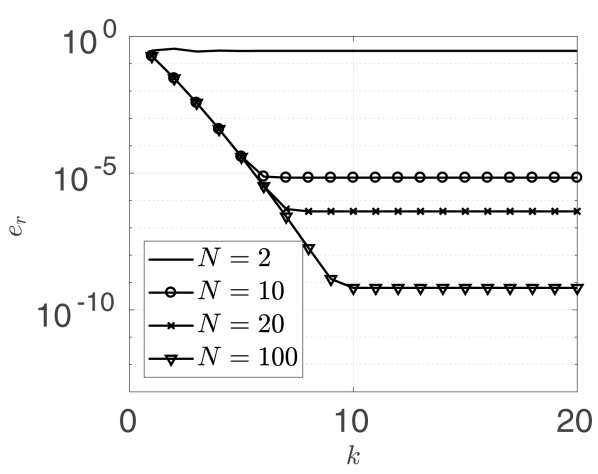

(c) Chebyshev

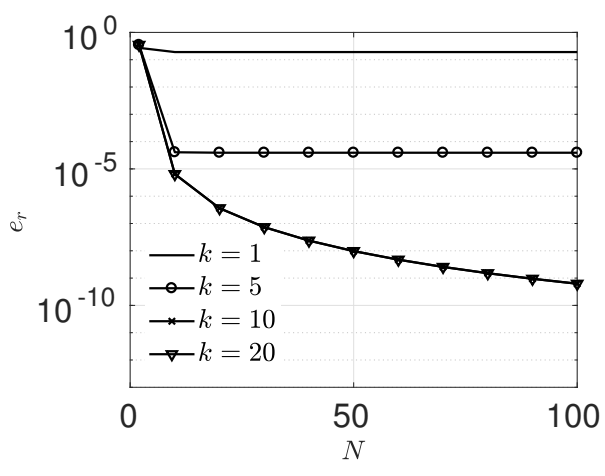

(b) Legendre

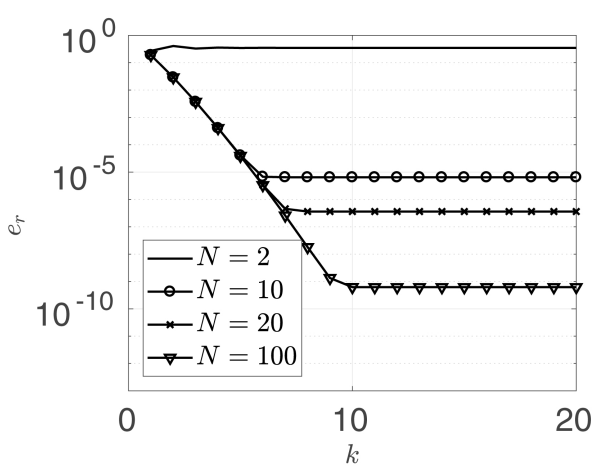

(d) Legendre

Figure 3: The relative error in terms of degree $N$ (a-b) and the number of iterations (c-d) corresponding to Example 2 for Chebyshev and Legendre bases.

\section{CONCLUSIONS}

This paper applies a numerical procedure for a nonlinear Volterra integral equations based on Chebyshev and Legendre bases functions considering the collocation method. The hypotheses of Theorem 3.1 are sufficient to conclude that the functional Volterra integral equation (1.1) has a unique solution in $L^{2}(a, b)$ space and all solutions of this equation are asymptotically stable. Moreover, examples show that our Theorem 5.1 was satisfactory to ensure the convergence of the collocation method for a moderate number of iterations.

Another important contribution is that we have been able to rigorously prove that the errors of approximate solutions decay exponentially in $L^{2}$-norm as the degree of approximation increases [15].

RESUMO. O método de colocação baseado em funções de base de Chebyshev, acoplado com processo iterativo de Picard, é proposto para resolver uma equação integral funcional de Volterra do segundo tipo. Usando o Teorema do Ponto Fixo de Banach, provamos teore- 
mas sobre a solução de existência e unicidade na norma $L^{2}$. Também fornecemos a análise de convergência e estabilidade do método proposto, o qual indica que os erros numéricos na norma $L^{2}$ decaem exponencialmente, desde que o kernel seja suficientemente suave. Resultados numéricos são apresentados e confirmam a previsão teórica da taxa exponencial de convergência.

Palavras-chave: método de colocação de equação integral funcional de Volterra, iteração de Picard.

\section{REFERENCES}

[1] H. Ansari \& P. Mokhtary. Computational Legendre Tau method for Volterra Hammerstein pantograph integral equations. B. Iran. Math. Soc., (2018), 1-19.

[2] J.S. Azevedo, S.P. Oliveira \& A.M. Rocha. Spectral element approximation of functional integral equations. Electron. T. Numer. Ana., 8(2) (2020), 164-180.

[3] C. Canuto, M.Y. Hussaini, A. Quarteroni \& T.A. Zang. Spectral methods. Scientific computation. In "Fundamentals in Single Domains". Springer-Verlag Berlin (2006).

[4] T.A. Driscoll. Automatic spectral collocation for integral, integro-differential, and integrally reformulated differential equations. J. Comput. Phys., 229(17) (2010), 5980-5998.

[5] N. Ebrahimi \& J. Rashidinia. Collocation method for linear and nonlinear Fredholm and Volterra integral equations. Appl. Math. Comput., 270 (2015), 156-164.

[6] D. Elliott. A Chebyshev series method for the numerical solution of Fredholm integral equations. Comput J., 6(1) (1963), 102-112.

[7] D. Henry. "Geometric theory of semilinear parabolic equations", volume 840. Springer (2006).

[8] A. Karoui \& A. Jawahdou. Existence and Approximate $L^{p}$ and Continuous Solutions of Nonlinear Integral Equations of the Hammerstein and Volterra Types. Appl. Math. Comp., 216(7) (2010), 20772091.

[9] A. Latif. Banach Contraction Principle and Its Generalizations. Topics in Fixed Point Theory, (2014), $33-64$.

[10] J. Liang, S.H. Yan, R.P. Agarwal \& T.W. Huang. Integral solution of a class of nonlinear integral equations. Appl. Math. Comput., 219(10) (2013), 4950-4957.

[11] L. Liu, F. Guo, C. Wu \& Y. Wu. Existence theorems of global solutions for nonlinear Volterra type integral equations in Banach spaces. J. Math. Anal. Appl., 309(2) (2005), 638-649.

[12] K. Maleknejad, K. Nouri \& R. Mollapourasl. Existence of solutions for some nonlinear integral equations. Commun. Nonlinear Sci., 14(6) (2009), 2559-2564.

[13] M. Meehan \& D. O'Regan. Existence theory for nonlinear Volterra integrodifferential and integral equations. Nonlinear Analysis: Theory, Methods \& Applications, 31(3) (1998), 317-341. 
[14] A.M. Rocha, J.S. Azevedo, S.P. Oliveira \& M.R. Correa. Numerical analysis of a collocation method for functional integral equations. Appl. Numer. Math., 134 (2018), 31 - 45.

[15] S. Shahmorad. Numerical solution of the general form linear Fredholm-Volterra integro-differential equations by the Tau method with an error estimation. Appl. Math. Comput., 167(2) (2005), 14181429.

[16] C.T. Sheng, Z.Q. Wang \& B.Y. Guo. An hp-spectral collocation method for nonlinear Volterra functional integro-differential equations with delays. Appl. Numer. Math., 105 (2016), 1-24.

[17] H. Tian. "Spectral methods for Volterra integral equations". Ph.D. thesis, Theses (Dept. of Mathematics and Statistics)/Simon Fraser University (1995).

[18] D. Trif \& C. Ionescu. Matrix based operatorial approach to differential and integral problems. In "MATLAB-A Ubiquitous Tool for the Practical Engineer". IntechOpen (2011).

[19] Z. Xie, X. Li \& T. Tang. Convergence analysis of spectral Galerkin methods for Volterra type integral equations. J. Sci. Comput., 53(2) (2012), 414-434.

[20] Y. Yang \& Y. Chen. Jacobi Spectral Galerkin and Iterated Methods for Nonlinear Volterra Integral Equation. J. Comput. Nonlinear Dynam., 11(4) (2016), 041027.

[21] Y. Yang, Y. Chen, Y. Huang \& W. Yang. Convergence analysis of Legendre-collocation methods for nonlinear Volterra type integro equations. Adv. Appl. Math. Mech., 7(1) (2015), 74-88. 